\title{
A NOVEL 3D MICRO MEMBRANE FILTRATION DEVICE FOR CAPTURE VIABLE RARE CIRCULATING TUMOR CELLS FROM WHOLE BLOOD
}

\author{
Siyang Zheng ${ }^{1}$, Henry K. Lin' ${ }^{2}$, Richard J. Cote ${ }^{2}$, and Yu-Chong Tai ${ }^{1}$ \\ ${ }^{1}$ Department of Electrical Engineering, California Institute of Technology, Pasadena, California, USA \\ ${ }^{2}$ Department of Pathology, University of Southern California, Los Angeles, California, USA
}

\begin{abstract}
Cancer is one of the most serious diseases facing human being. Improvement on cancer diagnostics has proved to be important for the outcome of cancer patients. We report here a novel micro membrane filtration device that captures rare circulating tumor cells from whole blood for early cancer diagnostics and treatment monitoring. The device is fabricated with multi layers of polymer material parylene-C. Tested with the model system, the device can capture tumor cells from whole blood with $86.5 \%$ capture efficiency. Different from previous membrane filtration devices, the cells are alive up to at least two weeks after isolation.
\end{abstract}

\section{INTRODUCTION}

Cancer has become the leading cause of death for persons younger than eighty-five years old in US since 1999 [1]. Over $90 \%$ of the solid tumor patients are killed by secondary metastatic tumors. Unfortunately, the metastasis process remains a major challenge for basic cancer research [2]. Imaging has been the primary tool for tumor diagnostics, but it requires the tumor to reach certain size to be detectable. During the metastasis process, tumor cells from the primary tumor travels to distant sites through circulation system (bloodstream or lymphatic circulation). Circulating tumor cells (CTC) are tumor cells shed into bloodstream during metastasis. If these CTCs can be detected, it can provide an invaluable tool for cancer early diagnostics and patient treatment monitoring. Technical difficulties for CTC detection include extremely low concentration (e.g., $\sim 1 / \mathrm{mL}$ or $1 / 10^{10}$ blood cells), and thus large sample volume for micro devices ( $\sim 7.5 \mathrm{~mL}$ whole blood). Therefore, a sensitive, efficient, and fast method for the separation and detection of CTCs at low cost is highly desirable and such a method may revolutionize the cancer diagnostics and prognostics [3-5].

Current clinical practice of CTC detection is primary based on gradient centrifugation [6]. A centrifugation reagent Ficoll-pague, which has specific gravity matched to mononuclear cells, is mixed with whole blood and centrifuged. The cell fraction including most of the lymphocytes and CTCs is extracted and immunohistochemically stained before laid on tens of glass slides. It can take a pathologist hours to read the slides. The only FDA approved automated system for CTC detection is based on immunomagnetic separation $[7,8]$. Antibody conjugated magnetic beads capture CTCs while other blood cells are flushed away. The assay and the instrument are very expensive and the fundamental problem of variable antigen expression on CTC surface limits the capture efficiency. Recently an immunoaffinity based "CTC chip" [4], where CTCs are captured with antibodies conjugated to device surface, has successfully demonstrated high enrichment CTC detection with patent samples from multiple metastatic cancers. While viable CTCs with high purity can be obtained, the capture efficiency and throughput need to be improved.

Previously, we reported a single-layered parylene membrane filter device that can capture CTCs from $7.5 \mathrm{~mL}$ of whole blood with $\sim 90 \%$ efficiency and $10^{7}$ enrichment [9]. Our filter utilizes size-based filtration because CTCs $(15-30 \mu \mathrm{m}$ in diameter) are significantly larger than erythrocytes $(6-9 \mu \mathrm{m})$ and leukocytes $(5-16 \mu \mathrm{m})$. However, the blood samples needs to be fixed (i.e., hardened) before filtration. Without fixation, cell membrane of CTC is ruptured during the filtration process. Fixation not only lengthens the sample preparation procedure and increases the back pressure, but, more importantly, kills the tumor cells, which limits further study of these rare cells. In this paper, we present a new 3D filter device that eliminates the need of blood fixation and reduces the shear stress exerted on the tumor cells during the filtration process. Experimentally, CTCs captured by the new device can stay alive for at least two weeks.

\section{DESIGN AND FABRICATION}

Parylene-C (poly-para-xylylene) is an ideal building material for this filtration device because of its unique properties. It is a mechanically strong (Young's modulus 4GPa and tensile strength: $70 \mathrm{MPa}$ ) while malleable (elongation to break $200 \%$ ) polymer. It is optically transparent in visible range, which facilitates direct pathological observation of stained cells under microscope. Chemically, it is inert to most of the chemicals and solvents used in standard chemical and biological laboratories, so all the post-processing (e.g. cell fixation, cell lysing, cell staining) can be performed after cell capture on devices. Parylene is a highly biocompatible polymer and can meet the highest standard for long-term implantation. Parylene membrane filtration devices will experience less clogging and membrane fouling due to reduced protein absorption and cell adhesion. Finally, high quality pin-hole free parylene thin film can be obtained with room-temperature chemical vapor deposition (CVD), and further patterned with standard lithography and oxygen plasma in a reactive ion etcher (RIE).

The fabrication started with $1 \mu \mathrm{m}$ thermal oxidation of prime $\mathrm{Si}$ wafer (Fig.1). The backside oxide was patterned and the front-side was stripped. A $5 \mu \mathrm{m}$ parylene-C thin film was deposited on front-side, and then melted at $350^{\circ} \mathrm{C}$. TMAH etching from backside made nine through-wafer cavities on each of the $1 \times 1 \mathrm{~cm}^{2}$ die, with silicon dioxide as etching mask and molten parylene as front-side protection layer. A $2.5 \mu \mathrm{m}$ second parylene layer was deposited from both sides and patterned with oxygen plasma. On the cavity regions, this second parylene layer sandwiched with the molten parylene layer and formed the bottom filter layer of the membrane filtration device. Then $6.5 \mu \mathrm{m}$-thick sacrificial photoresist was spin-coated and patterned, followed by $10 \mu \mathrm{m}$ parylene deposition and patterning from front-side. This is the top filter layer of the membrane filtration device. Finally sacrificial photoresist was dissolved in acetone and all the parylene layers were annealed at $190^{\circ} \mathrm{C}$ for forty-eight hours to strengthen the overall structure. The pore size, shape and density are defined by the photolithography process. The gap between the top and bottom membranes is defined by the thickness of the sacrificial photoresist. All these parameters can be controlled by design.

To keep device integrity during filtration, within each cavity there are 99 hexagonal patches (Fig.2). The top and bottom parylene membrane layers are in contact and annealed at the regions between the patches. Each patch has thirty-six $9 \mu \mathrm{m}$ diameter pores on the top parylene membrane and thirty-seven $8 \mu \mathrm{m}$ diameter pores on the bottom parylene membrane (Fig.3). The pore positions on the top and bottom membranes are shifted so that a 


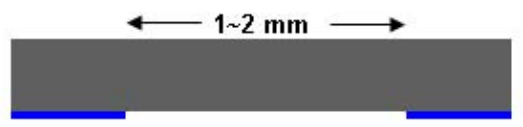

1. Thermal oxidation. Backside oxide patterning. Frontside oxide removal.

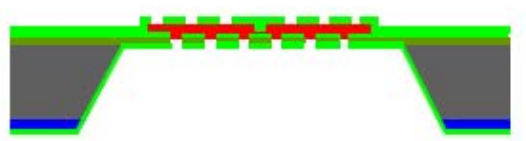

4. Sacrificial photoresist patterning.

$3^{\text {rd }}$ parylene deposition and patterning.

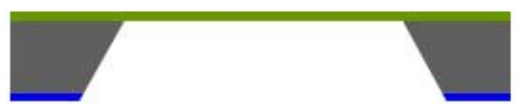

2. $1^{\text {st }}$ parylene deposition. Parylene melting. TMAH e tching for backside cavity.

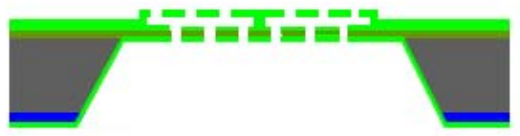

5. Sacrificial photoresist releasing. Parylene anuealing.

Figure 1: Process flow.

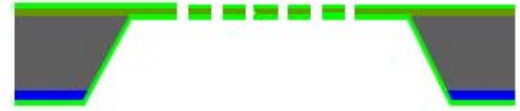

3. $2^{\text {nd }}$ parylene deposition and patterning.
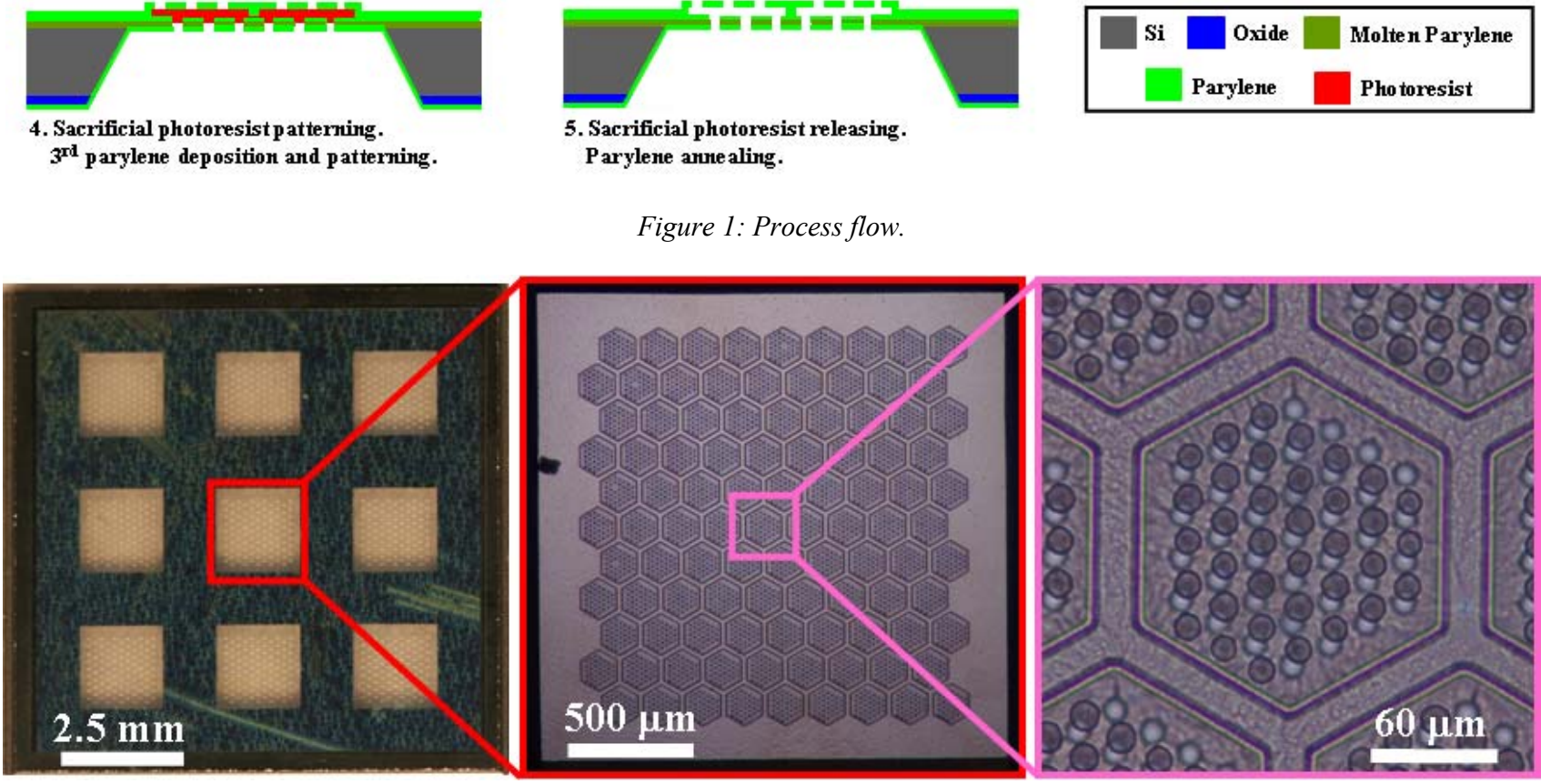

Figure 2: Fabricated device pictures. Left: each device has a 3x3 array of Si cavities. Middle: each cavity has 99 hexagonal patches. Right: each patch contains two layers of parylene filters with shifted pore positions. The gap between the two layers is $6.5 \mu \mathrm{m}$ and acts as the $3 \mathrm{rd}$ filtration dimension.

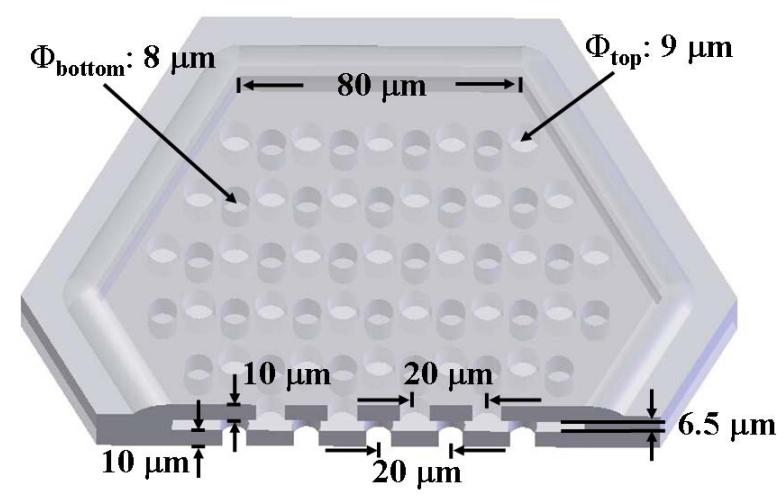

Figure 3: Device design showing the geometry of a single patch.

\section{EXPERIMENTAL}

Human breast adenocarcinoma cancer cell line MCF-7 is treated with green fluorescent stain carboxyfluorescein diacetate succinimidyl ester (CFSE), centrifuged and then resuspended in phosphate buffered saline (PBS) before testing. Live cells with intact cell membrane can keep CFSE inside and thus fluoresce, while CFSE leaks out in dead cells. CFSE stained MCF-7 cells in the stock solution are counted manually with a hemacytometer under an epi fluorescent microscope with a blue excitation green emission filter block (Nikon B-2E/C). The cells are diluted with PBS to the desired concentration before use. Cells are cultured in standard cell culture facility at $37^{\circ} \mathrm{C}$ and $5 \% \mathrm{CO}_{2}$. Dulbecco's modified eagle's medium (DMEM) with $10 \%$ fetal calf serum (FCS) is used as cell culture media.

For testing, a jig was made by two acrylic pieces with central holes (Fig.4). Four pieces of PDMS were used to sandwich the chip to form a top and a bottom chamber and as the sealing material to prevent leakage. The acrylic pieces and the PDMS pieces are clamped together. Sample is loaded into a syringe and the tip is inserted into a matching hole on the top PDMS piece. The sample is hand-pushed through the device. The back pressure experienced during filtration is normally very low.

\section{RESULTS AND DISCUSSION}

For the previous one layer parylene membrane filter, the cells have to be fixed before applying to the devices. Fixation hardens the cells by chemically crosslink cellular proteins. Without fixation, the cells are disintegrated during the filtration process due to the mechanical shear stress acting on the cell membrane during the filtration process (Fig.5A and Fig.5B). The 3D micro filtration devices have two layers of membrane filters and the pore positions on the bottom membrane are shifted from those on the top membrane. The cells are trapped in the pores of the top membrane 
and the bottom membrane provides mechanical support to the trapped cells. In this way, the shear stress exerted on cell membrane during the filtration process is minimized. As a result, in contrast to the faint green "ghost rings" on previous single-layered filter, the CFSE labeled MCF-7 cells show bright green fluorescence on the 3D microdevices after filtration, meaning the cell membrane is well preserved (Fig.5C).
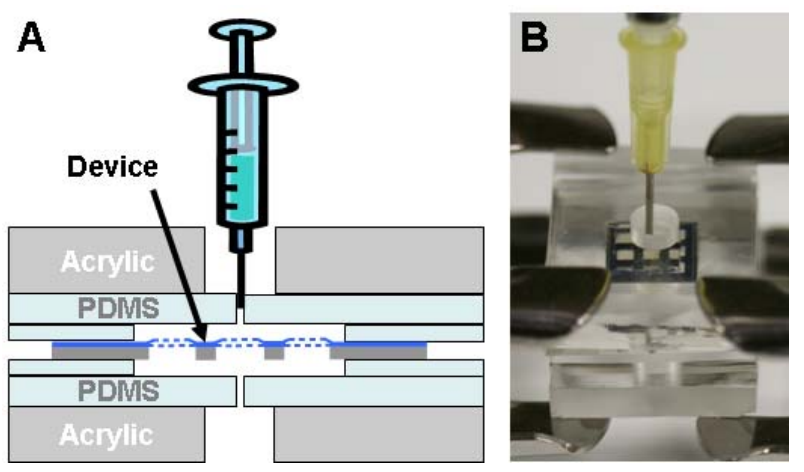

Figure 4: Device assembled for testing.
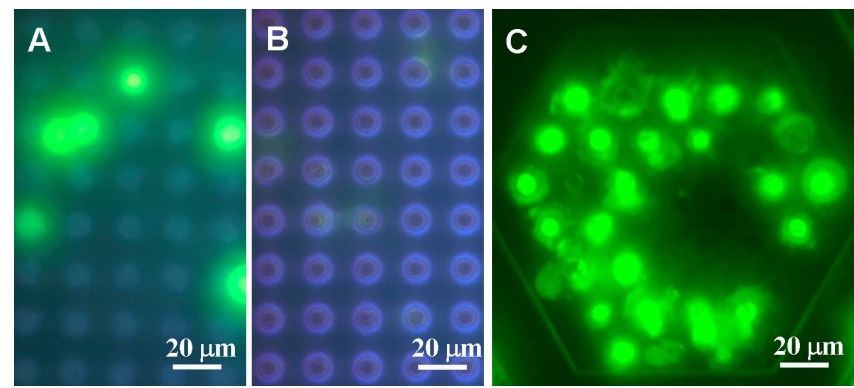

Figure 5: Experiments with MCF-7 tumor cells. A: positive control, CFSE labeled MCF-7 cells loaded on top a single layered parylene filter without filtering through. B: CFSE labeled MCF-7 cells filtered through a single layered parylene filter. The image is over exposed to show the faint green color around the pore edge. C: CFSE labeled MCF-7 cells filtered through a $3 D$ filtration device.

Capture efficiency is an important parameter for CTC isolation and we characterized it with a model system. The model system is constructed by spiking a known number of CFSE labeled human breast cancer cell line MCF-7 cells into dilute whole blood. In the results shown in Table 1, MCF-7 cells were counted manually under hemacytometer for five times. $342 \pm 58 \mathrm{MCF}-7$ cells were then spiked in $1 \mathrm{~mL}$ ten times diluted whole blood, and passed through the devices. Cells are counted under fluorescent microscope right after filtration. The MCF-7 cells are green fluorescent because of CFSE labeling while blood cells do not fluoresce. Capture efficiency is measured to be $86.5 \pm 16.5 \%$ with the model system, which is comparable to that of the previous single layer membrane filter $(\sim 90 \%)$.

To study the viability of the captured tumor cells, the devices were taken out from the jig assembly and put immediately into a petri dish filled with cell culture media and kept in cell culture incubator for further observation. After seven days in cell culture media, over $80 \%$ of the tumor cells still fluoresce brightly (Fig.6). In spite of the overwhelming number of blood cells around, the cellular membrane of these tumor cells is intact. It's interesting to note some of bright spots, which presumably are viable cells right after filtration, are missing after seven days. In three of these cases in
Fig.6, the cells are actually on the surface of the top filter, instead of being trapped in pores. So it is likely that these three cells detached from the device surface and moved into cell culture media during cell culture or device manipulation after filtration. For the rest nine cases where the cells seem to be trapped in pores of the top membrane on the first day but missing after seven days, there might be two possibilities. These cells might die during the seven day period and thus the CFSE dye leaks out, making them not fluoresce. Or these cells might migrate out of the pores. In fact tumor cells are famous for their ability to migrate during the metastasis process and this ability is an important characteristic of a metastatic tumor cell.

Table 1: Capture efficiency measurement.

\begin{tabular}{c|c}
\hline Experiment Number & Tumor Cell Count \\
\hline 1 & 288 \\
\hline 2 & 317 \\
\hline 3 & 281 \\
\hline 4 & 299 \\
\hline Average & $296 \pm 16$ \\
\hline
\end{tabular}

To further confirm the viability of the captured tumor cells, red fluorescent stain $\mathrm{C}_{12}$-resazurin (dodecyl-resazurin) purchased from Invitrogen, CA, USA is used to measure the metabolic level inside cells. $C_{12}$-resazurin is readily diffused into cell through cellular membrane. In metabolically active cells, non-fluorescent $\mathrm{C}_{12}$-resazurin is reduced to red-fluorescent $\mathrm{C}_{12}$-resorufin by some cellular enzymes. To use $\mathrm{C}_{12}$-resazurin for cell viability test, green fluorescent dye CFSE labeled MCF-7 cells were spiked into ten times dilute whole blood, and then filtered through a 3D filtration device. After checking the captured cells under epi fluorescent microscope with green emission filter, the device was kept in cell culture media for fourteen days. On the fifteenth day, $\mathrm{C}_{12}$-resazurin with a final concentration of $5 \mu \mathrm{M}$ was introduced to the device and the system was incubated in cell culture incubator for fifteen minutes. Then the device was taken out and observed under epi fluorescent microscope with green excitation red emission filter block (Nikon G-2E/C). Only metabolically active cells fluoresce red due to the reduction of C12-resazurin to red fluorescent $\mathrm{C}_{12}$-resorufin. As shown in Fig.7, all metabolic active cells including blood cells fluorescence red. Compared with the green fluorescent image which indicates positions of the CFSE labeled MCF-7 tumor cells only, almost all the tumor cells with complete cell membrane (green fluorescent) also show strong red fluorescent signals. Compared with green fluorescent image taken right after filtration, more than $70 \%$ of the CFSE labeled tumor cells are metabolically active after 14 days.

\section{CONCLUSION}

A novel 3D membrane filtration device was designed and fabricated with integrated parylene technology. The pore positions on the bottom membrane are shifted from the positions on the top membrane. The mechanical support provided by the bottom membrane to cells trapped in the pores of the top membrane effectively reduces the shear stress exerted on cell membrane. As a result, the tumor cells can be captured alive without any sample preparation other than dilution with PBS. The viability of the captured tumor cells are validated with two approaches. Green fluorescent dye CFSE assays for the integrity of the cell membrane, while red fluorescent labeling reagent $\mathrm{C}_{12}$-resazurin tests the cellular metabolic activity. In both cases, the results show at least $70 \%$ of the tumor cells isolated from the tumor cell spiked model 
system are alive for at least two weeks after filtration. The capture efficiency measured from the model system is $86.5 \%$, which is comparable to previous single layer parylene filter and higher than most of the other approaches for CTC isolation. The 3D micro filtration devices can be a valuable tool for metastasis research and clinical diagnostics of metastatic cancer.
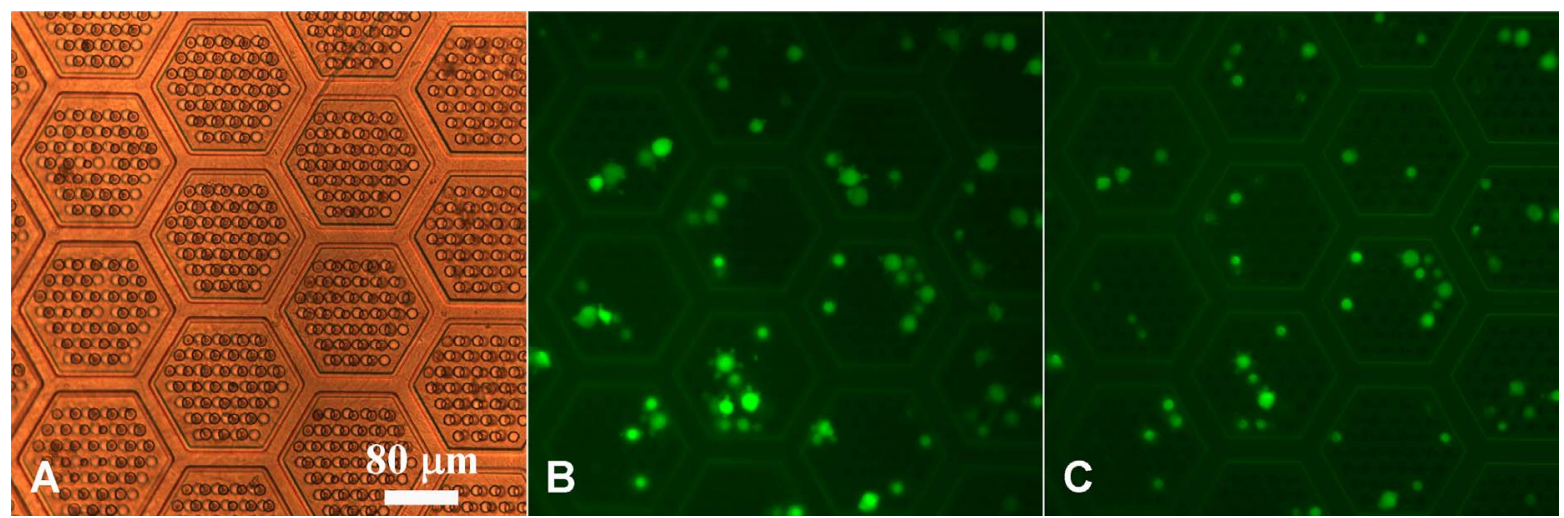

Figure 6: Experiments with the model system. CFSE labeled MCF-7 cells are mixed with ten times diluted whole blood and then passes through the $3 D$ filtration devices. A: Microscope image of the device after filtration under bright field illumination. B: Epi fluorescent image of the device right after the filtration. C: Epi fluorescent image of the device 7 days after the filtration.

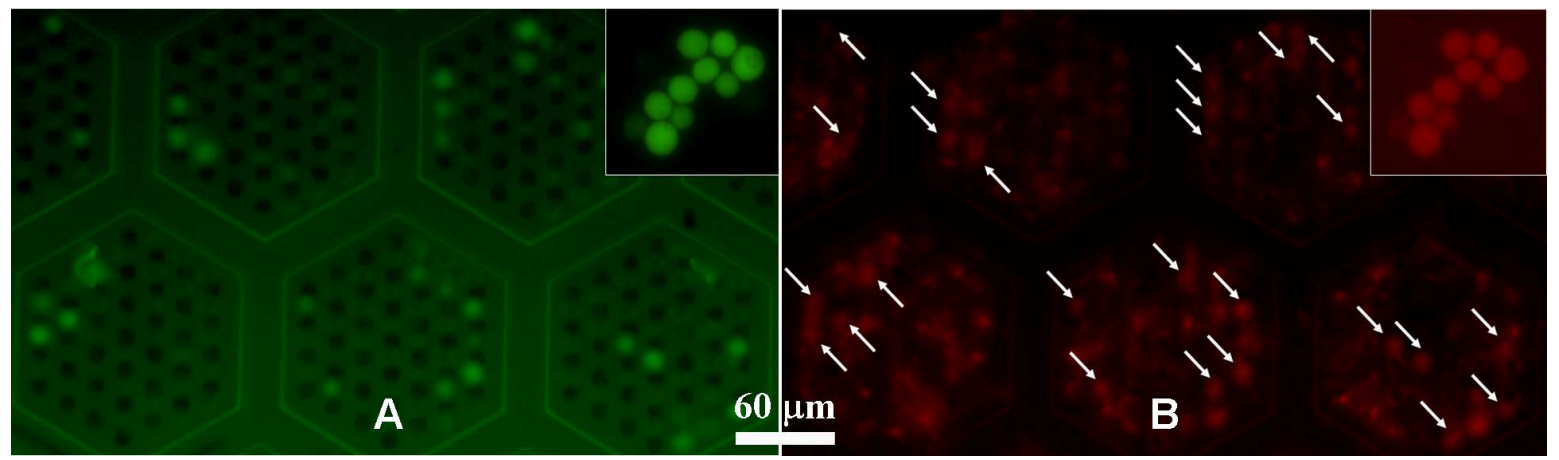

Figure 7: Cell viability tests. A: Epi fluorescent image (green channel) of a devicel4 days after filtered with the model system mixture. B: Epi fluorescent image (red channel) of same device stained with C12-resazurin at the 14th day. Arrows points to the positions of MCF-7 cells as indicated by figure 7A. Note metabolic active blood cells are also stained red. Insets are a positive control of MCF-7 cells 14 days after loaded onto a device without filtration.

\section{ACKNOWLEDGE}

The funding of the project is provided by NIH 1R21 CA123027-01. The authors would like to thank members of the Caltech Micromachining Laboratory for their valuable assistance.

\section{REFERENCES}

[1] A. Jemal, T. Murray, E. Ward, A. Samuels, R. C. Tiwari, A. Ghafoor, E. J. Feuer, and M. J. Thun, "Cancer Statistics, 2005," CA Cancer J Clin, vol. 55, pp. 10-30, January 1, 20052005.

[2] R. A. Weinberg, The biology of cancer. New York: Garland Science, Talyor \& Francis Group, LLC, 2007.

[3] G. P. Gupta and J. Massague, "Cancer metastasis: Building a framework," Cell, vol. 127, pp. 679-695, Nov 172006.

[4] S. Nagrath, L. V. Sequist, S. Maheswaran, D. W. Bell, D. Irimia, L. Ulkus, M. R. Smith, E. L. Kwak, S. Digumarthy, A. Muzikansky, P. Ryan, U. J. Balis, R. G. Tompkins, D. A. Haber, and $\mathrm{M}$. Toner, "Isolation of rare circulating tumour cells in cancer patients by microchip technology," Nature, vol. 450, pp. 1235-U10, Dec 2007.

[5] K. Pantel and R. H. Brakenhoff, "Dissecting the metastatic cascade," Nature Reviews Cancer, vol. 4, pp. 448-456, Jun 2004.
[6] O. Lara, X. D. Tong, M. Zborowski, and J. J. Chalmers, "Enrichment of rare cancer cells through depletion of normal cells using density and flow-through, immunomagnetic cell separation," Experimental Hematology, vol. 32, pp. 891-904, Oct 2004.

[7] U. Bilkenroth, H. Taubert, D. Riemann, U. Rebmann, H. Heynemann, and A. Meye, "Detection and enrichment of disseminated renal carcinoma cells from peripheral blood by immunomagnetic cell separation," International Journal of Cancer, vol. 92, pp. 577-582, May 152001.

[8] E. Racila, D. Euhus, A. J. Weiss, C. Rao, J. McConnell, L. Terstappen, and J. W. Uhr, "Detection and characterization of carcinoma cells in the blood," Proceedings Of The National Academy Of Sciences Of The United States Of America, vol. 95, pp. 4589-4594, Apr 141998.

[9] S. Zheng, H. Lin, J.-Q. Liu, M. Balic, R. Datar, R. J. Cote, and Y.-C. Tai, "Membrane microfilter device for selective capture, electrolysis and genomic analysis of human circulating tumor cells," Journal of Chromatography A, vol. 1162, pp. 154-161, 2007. 